\title{
Taire la chose et dire les mots. Ou les avatars de l'innovation pédagogique en Éducation physique au XXe siècle en France
}

Jacques Gleyse

\section{(2) OpenEdition \\ Journals \\ Édition électronique \\ URL : http://journals.openedition.org/trema/1552 \\ DOI : $10.4000 /$ trema. 1552 \\ ISSN : 2107-0997 \\ Éditeur \\ Faculté d'Éducation de l'université de Montpellier}

\section{Édition imprimée}

Date de publication : 1 septembre 2003

Pagination : 65-85

ISSN : $1167-315 X$

\section{Référence électronique}

Jacques Gleyse, « Taire la chose et dire les mots. Ou les avatars de l'innovation pédagogique en Éducation physique au XXe siècle en France », Tréma [En ligne], 22 | 2003, mis en ligne le 13 octobre 2010, consulté le 19 avril 2019. URL : http://journals.openedition.org/trema/1552 ; DOI : 10.4000/ trema.1552

Ce document a été généré automatiquement le 19 avril 2019

Trema 


\title{
Taire la chose et dire les mots. Ou les avatars de l'innovation pédagogique en Éducation physique au XXe siècle en France
}

\author{
Jacques Gleyse
}

1 La discipline Éducation physique (désormais EP), si elle trouve ses racines les plus profondes dans l'Antiquité grecque et romaine, n'est dénommée ainsi qu'à la suite de la publication en 1762 d'un ouvrage du médecin suisse Jacques Ballexserd, Dissertation sur l'éducation physique des enfants. C'est l'année où paraît L'Émile de Rousseau (lui aussi Genevois). La

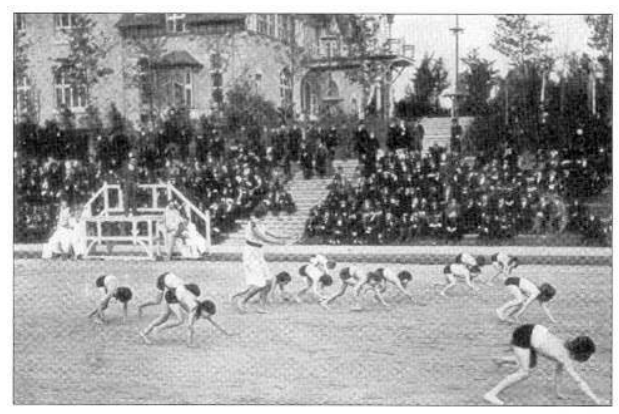
gymnastique avait déjà été remise au goût du jour (un peu dans la logique du néo-classicisme dans l'art) précédemment par le De Arte Gymnastica de Mercurialis en 1567, puis 1602, œuvre qui réalisait une compilation quasi exhaustive des pratiques antiques.

2 Rousseau affirmera avoir été plagié par Ballexserd, voire copié purement et simplement par lui. On comprend donc que c'est sur le modèle d'une «éducation négative » que la pratique EP s'instaure, autrement dit sur un modèle plus ou moins pédocentrique. En tout état de cause, La Dissertation de Jacques Ballexserd est avant tout une éducation globale des cinq sens, découpée au plan didactique en cinq catégories d'âge (à peu près les catégories wallonniennes ou piagétiennes).

3 En 1869, la Gymnastique trouve son premier programme et sa première obligation dans les lycées et collèges (c'est-à-dire qu'elle pourrait potentiellement toucher environ 100 000 élèves), mais sans aucun horaire obligatoire. C'est seulement en 1925 que deux heures 
hebdomadaires seront libérées dans l'emploi du temps de l'enseignement secondaire. L'EP, quant à elle, deviendra formellement obligatoire à l'école primaire avec les lois Ferry (entre 1880 et 1882).

4 C'est alors aussi à l'armée que la gymnastique deviendra obligatoire (pour les garçons naturellement). Le premier règlement militaire à s'intituler Règlement d'Éducation Physique y date du 21 Janvier 1910.

Mais, pour l'Instruction publique, c'est un texte paru au journal officiel du 11 Avril 1922 qui institue vraiment l'EP comme matière scolaire.

5 L'EP deviendra Éducation Physique et Sportive en 1943 sous le gouvernement de Vichy, et cette dénomination sera entérinée par les Instructions Officielles de 1945. Dès lors, la culture scolaire $d u$ corps est devenue une matière obligatoire dotée aujourd'hui d'horaires conséquents ( 3 heures dans le primaire, 4 heures en $6^{\mathrm{e}}, 3$ heures en $5^{\mathrm{e}}, 4^{\mathrm{e}}, 3^{\mathrm{e}}$ et 2 heures en seconde, première, terminale, voire 5 heures en terminale, sur option). Son coefficient au Brevet des collèges est de 1 , comme les autres matières, et 2 au baccalauréat.

6 Comme tout champ scolaire, celui de l'éducation physique a dû, sous l'action de nombreux acteurs, se constituer une didactique face aux transformations successives des modalités d'enseignement. Ces transformations ont souvent été considérées, dans un premier temps, comme des «innovations», puis assez rapidement d'autres « innovations » lui ont succédé.

7 Pourtant un constat doit être fait: si la plupart de ces innovations ont une base historique, elles sont aussi en rapport avec des conflits extrêmement puissants, voire fondamentaux en termes de valeurs. C'est cette base qu'il faut maintenant exposer, car elle permet de comprendre comment et pourquoi les mots changent mais les choses restent. C'est elle également qui donne intelligibilité à ce qui $a$ priori n'en a pas. C'est elle aussi qui permet d'analyser la dynamique d'une volonté constante d'innovation.

\section{Deux mystiques opposées à l'origine de deux didactiques}

8 En 1888, se créent, pratiquement en succession, deux associations qui vont être décisives pour la structuration du champ de l'EP et notamment pour la structuration de deux didactiques exogènes.

Le Comité pour la propagation des exercices physiques, dit " Jules Simon », du nom de son président (Pierre de Coubertin en est le très actif secrétaire général) est créé le $1^{\mathrm{er}}$ juin 1888. Il se donne pour but de favoriser la pratique de l'exercice physique, des jeux et des sports pour l'ensemble de la population française, et notamment dans les établissements scolaires secondaires.

On remarque tout de suite à la composition de celui-ci qu'il n'est pas du tout neutre au plan idéologique. I1 est, en effet, constitué d'une bonne part de notables réactionnaires ou conservateurs, de droite, et qui ont souvent soutenu Thiers. On y trouve bien sûr à sa tête Jules Simon, mais aussi Georges de $S^{t}$ Clair, Godard directeur de l'école Monge (catholique), le prince Georges Bibesco, Le vicomte de Janzé, Marcel Labbé, qui publiera le Traité d'EP de 1930 et sera un proche des Croix de Feu et eugéniste notoire, les généraux Barbe, Thomassin et Lewal (ancien ministre de la guerre, ayant participé à la répression de la Commune), Georges Picot, de l'Institut des sciences morales et politiques, Rieder 
directeur de l'École alsacienne... Son but est bien de propager l'exercice physique mais pas n'importe lequel. Il s'agit avant tout de faire prévaloir une pratique distinctive pour une élite aristocratique. Cette pratique c'est le Sport que Pierre Frédi, baron de Coubertin, définira très vite comme " la religion de l'excès ".

Face à ce camp se trouve d'abord celui des Sociétés de gymnastique de France créées le 28 septembre 1873. Son premier président Eugène Moïse Paz (1836-1901) est fils d'instituteur, professeur au Lycée Condorcet, ami d'Émile Zola, marqué à gauche, notamment, alors, par ses positions patriotiques. Il souhaite d'abord valoriser la pratique physique des plus faibles et du plus grand nombre. C'est de cette définition axiologique que naitront un peu plus tard deux autres associations plus ou moins filles de la première. On remarquera qu'elles seront créées en réaction à la mise en place du Comité pour la propagation des exercices physiques «Jules Simon » qui a avant tout pour but comme on l'a dit de développer un sport d'élite en dépit de certains propos démagogiques.

11 La Ligue Nationale d'éducation physique, de Paschal Grous-set (1844-1909) - alias Philippe Daryl - et sa copie locale la Ligue Girondine d'éducation physique de Philippe Tissié, sont créées respectivement les 14 octobre et 21 décembre 1888. Si la deuxième, provinciale, s'inscrit bien dans le même système de valeur que la première, elle n'en est finalement que la copie locale. Ici il convient aussi de regarder la composition du comité directeur originel. Il comprend des personnalités républicaines, de gauche, des francsmaçons, voire d'anciens communards et membres de l'Internationale. À sa tête est Marcellin Berthelot et l'on voit parmi ses membres: Georges Clémenceau, Jean Macé (fondateur de la Ligue de l'enseignement et franc-maçon), Alexandre Dumas, Michel Bréal (proche de Jules Ferry), Ferdinand Buisson (directeur de l'enseignement primaire de Jules Ferry et auteur d'un monument pédagogique républicain le Dictionnaire de pédagogie et d'instruction primaire), Fernand Lagrange, Étienne-Jules Marey, Pasteur, Millerand, Octave Gréard (autre proche de Jules Ferry), Jules Verne, Jean Richepin... Paschal Grousset, son président lui-même, d'abord blanquiste, fut député du $\mathrm{X}^{\mathrm{e}}$ arrondissement de Paris chargé des Relations extérieures de la Commune. Il échappa de peu à la Semaine sanglante et fut déporté en Nouvelle-Calédonie en compagnie de Louise Michel. Franc-maçon à son retour, il sera longtemps député socialiste indépendant de Paris. Il reviendra ensuite en Europe, suite à une évasion rocambolesque qui le mènera à l'exil en Angleterre, jusqu'à l'amnistie des Communards en 1880. Il écrira de ce fait en tant que journaliste au Temps sous le pseudonyme de Philippe Daryl, et en tant que romancier sous celui d'André Laurie.

12 On comprend bien que tout oppose Paschal Grousset et Jules Simon. Il y a d'abord entre eux des engagements opposés, mais aussi une idéologie. L'un est, pour le dire comme Michel Onfray ${ }^{1}$, dans une mystique de " gauche ", c'est-à-dire tourné vers le plus faible, le plus grand nombre, le droit humain, l'autre dans une mystique de "droite", plutôt tourné vers l'élite et le droit divin. On comprend donc que La Ligue française d'Éducation physique, prône l'exercice physique pour tous les enfants des écoles sous une forme relativement douce, alors que le Comité dit "Jules Simon» est partisan d'un sport distinctif d'élite (bien qu'il veuille favoriser l'exercice pour tous).

On comprend aussi que la Ligue soit créée contrele Comité quelques mois après lui en forme de résistance. Il est aussi possible d'en déduire que ces deux systèmes de valeurs antagonistes n'ont pas fini de s'affronter dans le champ de l'Exercice scolaire du corps. On se doute aussi que les didactiques qui découleront de ces deux visions du monde seront assez différentes. 


\section{Le sport contre l'éducation physique} en difficulté. tout aussi clair : conciliables. ouvrages :

La Ligue a, contrairement au Comité, fait sa place dans l'école républicaine de Jules Ferry, notamment par le biais de Philippe Tissié (qui a créé la Ligue Girondine) et d'un autre auteur important, secrétaire de la plupart des commissions ministérielles consacrées à l'EP : Georges Demenÿ (1850-1917), dont les positions sont très proches de celles de Paschal Grousset. Ce dernier, en outre est écouté, comme on l'a vu, par Ferdinand Buisson, ou Octave Gréard. Il fera accepter ses visions du corps dans l'école de la Troisième République. Il en ira autrement dans les lycées et les collèges, qui excluent les enfants du peuple, où le Comité règne.

Dans l'enseignement primaire et primaire supérieur, c'est en tout cas très clairement le parti de la Ligue qui est pris : une pratique médiane, pour les plus faibles, d'abord, et le plus grand nombre. L'EP s'oppose donc au sport par son intérêt pour la masse et les plus

Georges Demenÿ résume parfaitement cela, en 1902 :

"L'Éducation physique s'adresse à tous aux faibles surtout. Les résultats extraordinaires auxquels atteignent certains sujets d'élite frappent l'imagination, on admire le développement musculaire excessif, l'adresse, l'audace poussées jusqu'à la témérité. Mais on oublie que c'est là une exception.

Sans prétendre à de tels avantages tous peuvent s'améliorer... [...] Il est urgent de relever le niveau moyen d'une nation au lieu de chercher à produire quelques sujets hors pair $»^{2}$.

Philippe Tissié, comme on l'a vu, créateur de la Ligue Girondine d'Éducation physique, est

"On sacrifiait la réalité à l'apparence, et [...] sous prétexte d'entraînement, l'institution des records et des championnats avait pour effet d'exalter la force de quelques rares sujets spécialement organisés pour ces sortes de concours, mais d'éliminer la masse de tous ceux qui auraient dû bénéficier d'une éducation mieux comprise et surtout mieux appliquée. Une des causes du piétinement [...] était donc l'installation de records et de championnats dans les concours de gymnastique aux agrès, dans les jeux, dans les sports. Toute méthode qui, dans l'ordre physique et dans l'ordre intellectuel, s'applique à faire prévaloir les forts au détriment des faibles par la surenchère contribue à diviser les citoyens d'un même pays [...] Le 〈phénoménisme` empêche de penser [...] la collectivité est sacrifiée à l'individualité par paresse d'esprit $»^{3}$.

On voit comment, au début du siècle, le sport promu par le Comité est reçu par les plus fervents partisans de l'EP scolaire. C'est bien en effet sur la base de valeurs politiques, voire d'idéologie que s'instaurent les différences entre deux formes de pratiques. Dans ces conditions, on serait bien en mal de dire qui est innovant dans le champ. Sont-ce les partisans de l'EP ou les thuriféraires du sport? Ce que l'on peut constater en tout cas c'est que deux visions s'opposent qui ne semblent pas idéologiquement et axiologiquement

Le secrétaire du Comité, Pierre de Coubertin, montre un positionnement évidemment très antagoniste. Dès 1888, dans L'Éducation en Angleterre, il promeut l'image et l'idée du self made man, combattant très vivement ce qui pourrait ressembler à l'intérêt général et à l'intérêt du plus grand nombre. Il promeut au contraire l'image de l'individualisme le plus total et, bien sûr, le struggle for life, élément idéologique qui meublera la plupart de ses 
"Vous n'y croyez pas aux carrières que l'on se fait soi-même parce que vous songez à ces premières bouffées d'air pur qui grisent le collégien rendu à la liberté [...] ce ne sont point des administrés et des subordonnés, mai s des hommes libres que nos maîtres doivent former [...] ce nivellement égalitaire [...] ne fait que porter au sommet tant de médiocrité. Dans l'éducation et même plus qu'ailleurs, il y a des inégalités nécessaires. Renonçons donc à cette dangereuse chimère d'une éducation égale pour tous. " ${ }^{4}$ plus fort, l'intérêt individuel contre l'intérêt du plus grand nombre. Le modèle des collèges d'Eton, Rugby, Oxford, Cambridge, n'est d'ailleurs pas du tout insignifiant puisque ces établissements scolarisent l'élite aristocratique anglaise et sont fondés sur une idéologie de droite. Coubertin ne dit mot évidemment des grammar schools ou des progressive schools qui scolarisent le peuple et sont conçues sur une autre logique que l'élitisme.

21 Le sport est, dans cette optique, l'activité qui, portant au sommet un champion, sera valorisée.

"Que le sport constitue une chance de succès dans le struggle for life et il s'imposera sans peine $\|^{5}$

Le baron de Coubertin défend, par là même, son idéologie qui promeut « le débrouillard ». C'est dans cette même logique qu'il tentera de valoriser de même une pratique corporelle où finalement la métaphore du Roi de droit divin sera très prégnante: on parlera d'ailleurs rapidement de «roi du sport». La devise des jeux olympiques formulée par le Père Didon, Citius, Aldus, Fortius, correspond, bien sûr, à ce système idéologique, tout autant que le rejet de la devise des Satyresde Juvénal mens sana in corpore sono, à laquelle il substitue mens fervida in corpore lacertoso, aphorisme, inscrit en filigrane sur la page de garde de ses Leçons de pédagogie sportive ${ }^{6}$.

23 Le modèle royal et catholique est aussi très apparent dans l'affirmation que "le sport est la religion de l'excès »'.

On retrouve cela également dans la condamnation des partisans d'une gymnastique pour tous, modérée et favorisant les plus faibles.

Mais d'autres idéologies de droite non-monarchistes, plutôt d'extrême droite, vont apparaitre chez de Coubertin, montrant bien son marquage politique et expliquant peutêtre aussi son adoubement des Jeux de Berlin en 1936, contre ceux, populaires, de Barcelone.

"Le sport c'est une école d'audace, d'énergie et de volonté persévérante. Par son essence, il tend vers l'excès; il lui faut des championnats et des records et c'est sa belle et loyale brutalité qui fait les peuples forts et sains. $»^{8}$

C'est la même idéologie qui le conduit à rejeter la présence des femmes aux Jeux olympiques jusqu'en 1928, ou à affirmer que la race blanche triomphera toujours de la race noire qui lui est inférieure.

Au-delà même de cette première mystique du dépassement, le baron de Coubertin invente la pyramide au sommet de laquelle se tient le record, analogie de l'État, de l'Ancien Régime, au sommet duquel se tient le monarque :

"Le sport est la religion de l'excès [...] L'idée de supprimer l'excès est une utopie de non sportifs. « Pour que cent se livrent à la culture physique, il faut que cinquante fassent du sport. Pour que cinquante fassent du sport, il faut que vingt se spécialisent. Pour que vingt se spécialisent, il faut que cinq se montrent capables de prouesses étonnantes '. Impossible de sortir de là tout s'enchaîne. C'est ainsi que le record se tient au sommet de l'édifice sportif. Résignez-vous donc, vous tous adeptes de l'utopie contre-nature 
de la modération à nous voir continuer de mettre en pratique la devise donnée par le père

Didon jadis à ses élèves et devenue celle de l'Olympisme, Citius, Aldus, Fortius "».

Simon, Paschal Grousset créera un contre-feu, avec la Ligue. On comprend mieux aussi son acharnement à promouvoir les jeux traditionnels français et à refuser l'entrée du sport dans les établissements scolaires, dès 1890, dans le journal Le Temps; ainsi écrit-il le 16 Février 1890, sous le pseudonyme de Philippe Daryl :

"Nous ne parlons pas des très sérieux inconvénients moraux que peut avoir cette misérable idée de sport, introduite dans les mœurs scolaires, du pari et des vices anglais venant à la suite. [...] il ne s'agit à aucun prix de semer dans nos lycées de la graine de bookmakers... Les chefs d'établissement feront donc sagement en fermant leur porte au sport comme ils la ferment au tabac, aux livres pornographiques et de refuser l'accès de leurs collèges aux hommes étrangers à l'Université, à son esprit et ses devoirs. $»^{10}$

La Ligue demeurera dominante dans le domaine de l'EP scolaire, au moins jusqu'en en 1938. À partir de cette période, le champ commencera à se redistribuer et viendra le temps de la croyance dans un sport innovation pédagogique. C'est ce que l'on étudiera plus loin. Mais, une autre forme d'innovation se fait jour en EP et plus largement au ministère de l'Instruction publique et des Beaux-Arts: l'introduction des méthodes actives.

\section{Le rousseauisme « innovation » de l'entre-deux- guerres?}

Les Instructions de 1923, en EP, s'inscrivent à la fois dans la logique définie dès l'origine par Jacques Ballexserd et dans celle de la Ligue Française d'Éducation Physique. Elles ne font aucune place au sport. Par contre, comme l'ensemble des instructions de 1923 destinées à l'enseignement primaire, elles se veulent innovantes en développant les méthodes actives.

dénomination de Jean-Jacques Rousseau par son seul prénom témoigne parfaitement de cette volonté de développer "l'école nouvelle » pour reprendre la formule de Roger Cousinet :

«Le programme du cours supérieur présente, par rapport à celui du cours moyen, deux nouveautés: ‘éducation des sens〉 au cours de promenades scolaires et 〈application des exercices à la vie courantes. Il s'agit de faire l'éducation de la vue, de l'ouie, des autres sens par les procédés que déjà recommandait Jean-Jacques, et que, de nos jours le scoutisme a très heureusement renouvelés et perfectionnés $»^{11}$

30 L'ensemble des instructions et non seulement la partie consacrée à l'EP proposent de substituer des méthodes actives aux seules méthodes transmissives.

"À l'enseignement par l'aspect, [...] forme intéressante de la méthode concrète qui n'a pas dit son dernier mot et que le cinématographe va renouveler, il faut superposer une autre forme de la même méthode, qui n'en est encore qu'à ses balbutiements mais qui décuplera l'efficacité de l'art pédagogique, l'enseignement par l'action $»^{12}$.

31 Évidemment, il y a un pas des textes officiels à la pratique pédagogique que ne sousestime pas Léon Bérard, signataire du texte, mais celui-ci croit aussi formuler une vision totalement innovante de l'éducation en général et de l'éducation physique en particulier. Or, des méthodes Montessori, Decroly, Dewey, des pratiques des Maîtres Camarades de Hambourg, à bien avant, l'éducation de Gargantua ou d'Émile (sans même parler de la maïeutique socratique), les pédagogies actives sont déjà connues depuis quelques 
décennies, voire quelques siècles. Certes elles se profilent pour la première fois dans le système de l'Instruction publique, seulement à ce moment là. Mais, on va voir que la redécouverte de cette «innovation" sera récurrente dans le champ de l'EP, et plus largement dans celui de l'éducation.

Ces Instructions de 1923 sont, en tout état de cause, la source à laquelle s'abreuveront nombre d'auteurs au cours du XX $X^{e}$ siècle en EP. Les clefs en sont :

- l'adaptation des exercices selon les âges des enfants (petits, moyens, grands), l'intérêt pour les plus faibles : " Tout enfant doit participer aux exercices [...] aucun ne peut être dispensé [...] trop souvent on dispense de ces exercices les enfants qui précisément en auraient le plus besoin, puisqu'ils sont les moins vigoureux et les plus maladifs »;

- la liberté de mouvement (p. 134);

- l'utilisation de pratiques ludiques («Au cours préparatoire les jeux prédominent »);

- le travail dans la joie et le plaisir : (« on ne travaille bien que dans la joie », "Le plaisir dont il s'agit n'est pas une jouissance passive, c'est la joie qui accompagne toute activité libre, consciente de travailler à la réalisation d'un bel idéal »);

- la relation pédagogique affectueuse : («Ce n'est pas par la crainte, c'est par l'affection que le maitre obtient le travail le plus régulier et le plus productif.»)

On comprend donc que le dernier paragraphe de ces Instructions puisse s'ouvrir sur la phrase suivante: "Plus d'air, plus d'aisance, plus de liberté, plus de joie et partant plus de travail. $»^{13}$

Tel est le fond global sur lequel se construira dès lors toute innovation en EP jusqu'à la fin du siècle. D'un côté, le pédocentrisme rousseauiste et de l'autre l'introduction de nouvelles pratiques. On voit que ce processus est construit d'une part sur une volonté ministérielle émergeant de positions républicaines (remontant à l'époque révolutionnaire), et d'autre part, un enjeu politique au travers des pratiques corporelles proposées.

\section{Les Bases Psychologiques de l'Éducation physique, première redécouverte du pédocentrisme}

De 1935 à 1941, Ernest Loisel (1882-1943) sera le deuxième directeur de l'École normale d'Éducation physique, (institution souhaitée par les républicains depuis près d'un siècle sur le modèle de l'École normale supérieure pour les autres disciplines). Il n'est pas professeur d'EP, mais agrégé de Lettres. Il a cependant été choisi par le ministre pour ses actions innovantes en la matière dans le cadre de sa fonction en tant qu'Inspecteur d'académie.

Il publie en 1935, année de sa nomination à la tête de cette institution souhaitée par les républicains depuis près d'un siècle, un ouvrage, qui sera encore réédité en 1955 en raison de la demande constante des enseignants et des étudiants: Les Bases psychologiques de l'Éducation Physique. Ce texte est bien sûr considéré comme particulièrement innovant en 1935 puisqu'il sera encore à l'honneur dans les années soixante. Or en fait de «bases psychologiques » il fait surtout l'histoire des méthodes actives d'éducation en général et de l'EP en particulier.

On peut résumer l'essentiel de sa pensée par cette phrase : «En Éducation physique, le jeu, le vrai jeu, c'est-à-dire l'action qui ne poursuit aucun but utile, a sa place marquée. Le professeur ne 
peut trouver que des avantages à suivre l'enfant dans l'évolution de ses goûts et de ses 〈instincts». » ${ }^{14}$ Et celle-ci de 1939 (reprise dans la revue EPSen 1954, op. cit.): «Ajoutons que l'enfant a raison et que le professeur a tort, car l'éducation se fait suivant l'ordre naturel et non l'ordre logique, ou plutôt l'ordre naturel précède l'ordre logique. La vie commande. C'est ce qu'enseigne la pédagogie moderne, l'école active; c'est ce que f.-J. Rousseau écrivait voilà près de 200 ans. $»^{15}$

Le seul plan de l'ouvrage Les bases psychologiques de l'Éducation physique, permet de comprendre la révolution que tente d'opérer l'auteur. La première partie est en effet consacrée dans sa totalité à « l'école active » et c'est bien cela qui est premier dans la pensée de Loisel. Sa référence en matière d'éducation se trouve essentiellement dans les œuvres de Rabelais ou de Rousseau; le corps est, dans cette optique, partie intégrante d'une éducation morale fondée sur les méthodes actives: "Dans le plan d'éducation intellectuelle, les besoins du corps restent secondaires, on ne leur fait une place qu'à regret. Dans le plan d'éducation active, le corps a sa place marquée. Il est en effet l'instrument de l'action ${ }^{16}$.

Cependant le deuxième directeur de l'ENEP est aussi lucide sur les chances de réussite de ses propositions au moment où il écrit : "Il est possible que la pédagogie active reste encore longtemps une utopie, que les contradictions qu'elle contient restent inconciliables, que les essais tentés partout soient sans lendemain. C'est pourtant dans son cadre que nous devons chercher une méthode d'éducation physique car elle seule permet à l'éducateur d'aujourd'hui de faire des hommes. $»^{17}$

Dès lors, la méthode (en termes de techniques corporelles cette fois) qui correspond le mieux aux visées de méthodes actives, c'est évidemment la "méthode naturelle", puisque c'est elle qui fait, selon lui, le plus de place à l'instinct, au respect de la nature corporelle. C'est elle qui prend le plus de distance avec des exercices, tels ceux proposés par les méthodes suédoise ou Demenÿ (méthodes de gymnastique construite du début du siècle), qu'il qualifie d'artificiels: "L'éducateur physique, se proposera essentiellement d'apprendre aux élèves à courir, à sauter, à grimper, à porter, à lancer, à enfoncer et à nager avec la conviction que tous les exercices qui n'ont pas pour but l'acquisition de ces mécanismes sont artificiels et arbitraires. ${ }^{18}$

41 Le vibrant hommage qui est rendu à Loisel par Yvonne Surrel directrice de l'Ecole normale de jeunes filles, en 1954, dans la revue EPS, ou celui de ses anciens élèves en 1955 lors de la réédition des Basespsychologiques de l'Éducation physique,témoigne de l'importance de sa pensée et de sa diffusion potentielle, surtout chez les enseignantes issues de l'ENSEP filles.

Pourtant, on voit bien que ce qui est considéré comme innovant, au sortir de la deuxième guerre mondiale, a déjà été l'objet de nombreux écrits au cours des décennies précédentes. Sans doute l'écrit n'est-il pas devenu acte (mais peut-il vraiment le devenir ? ). C'est peut-être cela qui explique la volonté insistante de percevoir ces propositions comme toujours neuves.

43 Il faut également redire qu'Ernest Loisel tente de promouvoir des techniques du corps qu'il juge innovantes : la méthode naturelle de Georges Hébert (marcher, courir, sauter, grimper, lever, lancer, porter, quadrupédie, se défendre et nager). Là encore, ce mouvement sera constant au cours du siècle. À peine une technique du corps est-elle installée qu'il convient d'en promouvoir une nouvelle, un peu comme si les contenus devaient en permanence se mettre en contact avec le monde social changeant ou avec les pratiques corporelles «à la mode ». On verra, qu'au cours de la deuxième moitié du XX 
siècle c'est, comme au tout début du XXe siècle, autour de l'introduction des techniques sportives que portera ce débat sur l'innovation. Rappelons toutefois ici que Georges Hébert est le rédacteur du Sport contre l'éducation physique, en 1925. On voit donc que le recours à sa méthode n'est pas neutre pour Ernest Loisel : il prend le parti, comme on l'a $\mathrm{vu}$, d'une vision de l'éducation physique républicaine face au sport aristocratique et monarchiste. Il opte pour une mystique de gauche face à une mystique de droite.

Les Instructions officielles relatives aux arrêtés du 23 mars 1938 et du 11 juillet 1938 cherchent à rendre effectives ces pratiques et ces idéologies.

"Les expériences faites depuis deux ans [il s'agit d'expérience pédagogiques valorisant le mi-temps pédagogique: éducation physique et sieste sont au programme de l'école avec les méthodes actives] ont suscité un grand mouvement d'intérêt. [...] De toutes ces tentatives que l'on groupe sous le nom d'École Nouvelle et qui visent à faire un appel direct à l'activité spontanée de l'enfant, nous avons beaucoup à tirer. Nous souhaitons que la curiosité de nos maîtres soit orientée dans ce sens. $\|^{19}$

On peut supposer que les pratiques n'avaient pas suivi les propositions, pourtant si valorisées, d'Ernest Loisel, puisque le législateur se croit tenu de les suggérer fortement de nouveau aux enseignants. On retrouve d'ailleurs encore dans ce texte la référence lancinante à Rousseau : "L'objection est que nous allons imposer un programme à ces exercices et que nous serons assez loin des formules qui font tout reposer sur le libre choix de l'enfant. Elle n'est pas nouvelle et les premiers critiques de l'Émile l'ont déjà faite. Mais toute éducation est suggestion et liberté ordonnée. ».

L'activité de l'enfant est de nouveau promue face à une pratique pédagogique qui ne la solliciterait pas assez: "L'enfant devient l'artisan de sa propre éducation en même temps que son sens social se développe. $\|^{20}$

On voit donc, là encore, dans l'éducation en général et dans le champ de l'EP en particulier, que la mise de «l'enfant au centre de l'éducation » promue par les lois Jospin en 1989, et que veut aujourd'hui remettre en cause Luc Ferry, est finalement une sorte d'éternel retour à l'instar de celui plus général de l'Histoire décrit par Vico en 1726 dans La Sciencia Nuova.

Et les Instructions officielles de 1923 ne se contentent pas de promouvoir les méthodes actives et le mi-temps pédagogique. Elles les mettent à l'emploi du temps : par semaine pour le cours supérieur, " 2 heures de dessin, 2 heures de travail manuel, 3 heures de sport et d'éducation physique, 3 heures d'activités dirigées et de chant et 1 heure de récréation "; horaires à peu près identiques pour les classes de fin d'études, et pour les écoles primaires en général (arrêté du 11 Juillet 1938).

L'EP trouve là un horaire conséquent (et sans doute très partiellement appliqué), mais on doit noter que ce n'est plus seulement d'EP dont qu'il est question mais aussi de Sport. On peut donc penser que le conflit du début du siècle opposant ces deux pratiques est en voie de règlement ou du moins qu'un métissage idéologique s'initie dans ce texte.

\section{L'Éducation nouvelle du corps : le sport?}

Comme on le sait le gouvernement du Front Populaire, par le biais du Brevet Sportif Populaire, cherchera à promouvoir le Sport pour tous. Ici, la volonté marquée est de permettre l'accès à une pratique, considérée jusqu'alors comme bourgeoise, à la très grande masse des élèves et plus largement de la population. Il s'agit de démocratiser le sport, devenu d'ailleurs, depuis les Jeux Olympiques de Berlin (et ceux Internationalistes 
de Barcelone, avortés à la suite de la Guerre d'Espagne) un enjeu de politique internationale. largement gagnés aux idées hébertistes, comme en témoignent notamment encore les Instructions officielles de 1941 (en partie rédigées par Ernest Loisel ${ }^{23}$ et toujours majoritairement proches de la Ligue Française d'Éducation Physique (qui a depuis 1888 connu plusieurs présidents de Philippe Tissié à Raoul Fournier), il convient d'abord de montrer la complémentarité du sport et de l'EP : «Afin de mettre un peu de clarté dans les esprits [...] faisons dès à présent une distinction entre le sport et l'Éducation physique en soulignant que les activités ne doivent pas être opposées l'une à l'autre. Elles se complètent : il n'y a pas d'Éducation physique sans sport et inversement [...] L'Éducation physique est l'ABC du sport. Sa valeur est surtout corporelle. "

53 La place est dès lors préparée pour un sport considéré comme le but ultime et le sommet de l'édifice EP. Poursuivre l'EP à partir d'un certain âge, ce serait continuer de balbutier à l'heure de la lecture des grands textes classiques. Pratiquer l'EP dans l'enseignement secondaire (ou primaire supérieur) serait poursuivre des pratiques de bébé à l'âge d'homme. Pourtant l'idéologie véhiculée par le sport est bien perçue par Maurice Baquet comme étant la domination du plus fort sur le plus faible :

«Le sport qui est synonyme de lutte, vise au maximum de rendement dans un effort individuel ou collectif par l'utilisation des qualités naturelles ou acquises à l'occasion de jeux ou d'exercices physiques impliquant l'idée de compétition. Le sport est une forme supérieure du jeu, c'est par le sport et la saine compétition que nous arriverons à conquérir la jeunesse à une activité susceptible de lui donner le goût de l'effort individuel pour un idéal collectif. $»^{24}$

Cela ne trouble pas l'auteur, et cela ne saurait le troubler. En effet, sa propre ascension sociale est due à la pratique sportive. Il est le modèle même du self made man promu par l'idéologie sportive. C'est par son combat contre les autres qu'il a pu arriver à la place qu'il occupe. Afin de diffuser l'idéologie dans la population, il s'agit donc de la faire adopter par toute la jeunesse et en fait de rejeter comme désuètes, passéistes, les autres formes de pratiques corporelles, de les disqualifier comme rétrogrades, voire obsolètes. C'est ce à quoi vont s'atteler dès lors certains enseignants de l'ENSEPS garçons (après 1946) et bon nombre de formateurs en EPS impliqués dans la Fédération Sportive et Gymnique du Travail, mais aussi le gouvernement gaulliste après 1958.

Pour les FSGTistes, l'option est claire : faire pratiquer le sport au plus grand nombre c'est transformer une activité réservée à l'élite bourgeoise en activité populaire de masse (l'analyse n'est peut-être pas faite de la finalité de cette activité : désigner une 
«musculocratie »). Pour le gouvernement gaulliste, il s'agira de recruter dans la masse des pratiquants des sportifs de haut niveau, susceptibles de promouvoir une politique de prestige de la France sur la scène internationale. Les professeurs d'EPS vont se laisser convaincre que le sport est une activité à la mode et moderne qui convient mieux aux élèves.

Le sport doit cependant pour obtenir ce statut être décrit comme une innovation technologique (alors que « to sport » - parier sur des chevaux - était utilisé en Angleterre au XVII ${ }^{e}$ et XVIII ${ }^{e}$ siècle et que la plupart des sports trouvent leurs origines dans des jeux traditionnels ruraux standardisés par l'International Board dès 1866 en Grande-Bretagne). Le sport doit être décrit comme innovant et moderne alors que l'EP, à l'inverse, doit être affublée de passéisme, de traditionalisme, d'anti-modernité, alors que nous avons vu que leur naissance est quasi-simultanée au plan institutionnel, mais que ce qui les distingue, c'est l'idéologie sur laquelle les deux pratiques reposent.

Le sport doit devenir l'emblème de la jeunesse : "Quoi qu'il en soit c'est par le sport et la saine compétition que nous arriverons à conquérir la jeunesse à une activité susceptible de lui donner le goût de l'effort individuel pour un idéal collectif. $»^{25} \mathrm{Car}$ le sport, c'est " de la morale en action " (nous avons vu quelle morale!).

Le sport, dès lors, pour un certain nombre d'acteurs du champ de l'EP, tout comme les méthodes actives, va être présenté comme innovant et surtout en adéquation avec la jeunesse.

Jacques de Rette, en juillet 1962, écrira, dans la revue EPS, (revue professionnelle qui paraît, depuis 1950), un texte intitulé «La gymnastique de grand-père est MORTE!!! » ${ }^{26}$ qui constitue en quelque sorte l'acmé de ce point de vue visant à discréditer définitivement tout ce qui n'est pas la méthode sportive. Le sport, dès lors, pourra être vu comme le seul facteur d'innovation (avec les méthodes actives). La dénomination de l'action est d'ailleurs suffisamment claire à ce sujet.

60 Un peu avant lui, Justin Tessié, longtemps formateur à l'ENSEPS garçons, propose un «Essai d'une systématique sportive » qui, lui aussi, décrit la pratique sportive comme une innovation: "En dehors du travail professionnel, le sport est devenu un facteur essentiel de l'activité physique de l'Homme moderne [...] Le sport expression sociale de l'éducation physique exerce une influence décisive sur les tendances et intérêts de l'enfant. ${ }^{27} T$ Tout est dit ou presque. Le sport est une innovation, le sport est moderne alors que l'EP est traditionnelle voire archaïque. Le sport doit donc prendre la plus grande place en EP, voire occuper toute la place.

\section{Quelques résistances sporadiques à «l'innovation » sportive}

61 En 1949, Pierre Seurin, membre et président de la Ligue Française d'Éducation physique, fait paraître un ouvrage qui, se vendant à plus de 12000 exemplaires, constituera une sorte de bestseller pour la profession des enseignants d'EPS et des instituteurs (entre 1949 et 1965). Celui-ci tente, encore là, comme Grousset, Tissié, Demenÿ ou Hébert, bien plus tôt, de montrer les facettes idéologiques du sport et sa potentielle nocivité morale. Mais la bataille semble perdue d'avance car la gymnastique et l'EP sont désormais sur une position défensive, en terme de "modernité » et d'innovation, ce qui ne permet pas de comprendre les différences fondamentales en termes d'axiologie : 
«Si l'on peut admettre que le but lointain du sport est d'ordre éducatif général, il faut convenir que ses moyens sont souvent différents de ceux de l'éducation physique.

» Le principe majeur du sport est l'effort maximum pour la victoire (sur l'obstacle, le temps, l'adversaire) et l'adaptation du sujet à des conditions indépendantes de lui (définies par des règlements). Techniquement donc et psychologiquement, l'éducateur qui prétend faire de l'éducation physique par le sport (et plus étroitement encore par le basket, le football, l'athlétisme même) s'emprisonne au point de départ dans une difficulté inutile.

"Pédagogiquement aussi il est faux de considérer un seul centre d'intérêt: la compétition, élément fondamental du sport. Pourquoi par exemple, l'entraide ne prendrait-elle pas autant d'importance que la compétition dans un système général d'éducation physique (une entraide qui n'oppose personne et non la seule coopération au sein d'un groupe pour vaincre un autre groupe). $»^{28}$

On voit pourtant que Pierre Seurin est cantonné dans une dynamique de résistance et non de propositions vues comme innovantes. Il tente de préserver l'intérêt de l'enfant et une vision pédagogique où la morale républicaine doit être première, face à l'idéologie du plus fort. Sa bataille est peut-être perdue d'avance puisque ce qu'il présente et représente ( $\mathrm{La}$ Ligue Nationale d'Éducation Physique) n'est pas perçu comme «moderne», ni " innovant», mais, au contraire, dans la mesure où le sport est, lui, décrit comme «moderne », par ses thuriféraires et sans doute déjà par leurs épigones à l'ENSEPS garçons, comme conservateur ou désuet.

On voit, en tout cas, que c'est toujours le même débat qui revient sous des formes reconsidérées. Si au début du siècle c'était plutôt les partisans de l'EP républicaine qui dominaient, il n'en va plus de même au cours des «Trente glorieuses". L'idéologie sportive l'a emporté en étant perçue comme moderniste et innovante. La domination du plus fort sur le plus faible est davantage valorisée (sans doute par le plus grand nombre), comme l'idéologie du self made man, que celle de l'intérêt pour le plus faible et le plus grand nombre. Cela peut d'ailleurs sans doute être compréhensible au regard du contexte social d'une croissance très dynamique où la promotion sociale est effective pour un grand nombre de personnes, et où « l'ascenseur social» fonctionne à plein régime (voir les travaux des économistes).

L'aboutissement de cette logique se trouve dans les Instructions officielles publiées le 19 octobre 1967 qui tentent de concilier les inconciliables au plan idéologique : le Sport et l'EP. Cette impossible conciliation se trouve dans l'appellation absconse au plan sociologique: Activités Physiques et Sportives (cherchant à travestir le mot «sport » et l'idéologie qu'il porte initialement) et dans le tableau à double entrée qui tente d'associer (à gauche du tableau : ce qui n'est sans doute pas un hasard) des « intentions éducatives » (c'est l'enfant qui est là au centre) avec (à droite du tableau) - très majoritairement des pratiques sportives (c'est la « matière » qui est ici première).

La colonisation (ou le métissage ?) du champ de l'EP par l'idéologie sportive est peut-être achevée. "On sait le développement considérable qu'a connu [...] le phénomène sportif tant à l'école que hors de celle-ci [...]. C'est la raison pour laquelle, parmi toutes les activités physiques, il doit, dans la majorité des cas, tenir la plus grande place $»^{29}$.

Le fait d'ailleurs que le sport soit considéré comme "dépourvu de finalités propres »", exprime assez bien la nécessite de dissimuler au regard d'un certain nombre d'enseignants, pour éviter toute résistance politique, l'aspect idéologique initial du phénomène sportif. Le sport est désormais moderne et innovant, l'EP rétrograde ou 
réactionnaire, ce qui est pour le moins paradoxal au regard de leurs origines idéologiques.

\section{Et ça continue encore et encore...}

67 À la suite, un autre conflit va éclater. La rhétorique et les fondements en demeurent identiques. Il oppose Jean Le Boulch à Robert Mérand et Monique Vial aux travers de deux organes: Les Cahiers Scientifiques d'Éducation physique et Les Cahiers du Centre d'Etudes et de Recherches Marxistes. Il trouve sa résolution provisoire, comme on l'a vu sous la forme d'un «non-dissensus $~^{31}$ constitué par les Instructions officielles de 1967. La publication de l'ouvrage de Jean Le Boulch en 1977 Face au sport ${ }^{32}$ en est le témoin privilégié pour la décennie suivante. Le Boulch face à cette vision dominante et à sa présentation comme moderniste, tente de montrer les limites éducatives du phénomène sportif et surtout de promouvoir une vision "scientifique", moderniste, de l'EP ou plutôt selon son expression, de « la psychocinétique ».

Dès ce conflit enterré et dès lors que la didactique du sport est devenue le paradigme dominant en EP, un nouveau débat anime le territoire de l'Éducation physique et sportive : pédagogie par objectifs ou pédagogie des conduites motrices ou du sens? Ici, s'affrontent, souvent paradoxalement, les précédents partisans de la didactique du sport (proches de la FSGT, plutôt proches du Parti Communiste) et notamment certains dirigeants du SNEP (plutôt la tendance Unité Action, alors au sein de la FEN) et un nombre non-négligeable d'acteurs dans la mouvance du SGEN CFDT (plutôt socialistes), de l'École Émancipée (Tendance de la FEN, plutôt extrême gauche) et de la revue Les Cahiers Pédagogiques (plutôt tendance UID de la FEN), ou encore de la revue Quel Corps? (Plutôt extrême gauche). L'Éducation physique et sportive, la «didactique des APS » est remise en cause au profit d'une pédagogie des «conduites motrices» et des «maîtrises de l'exécution ».

69 En réalité, la « didactique des A.P.S. » est une formule qui se présente comme innovante alors qu'elle n'est finalement qu'un prolongement des positions du Baron Pierre de Coubertin ou de Maurice Baquet, visant à faire primer les techniques et l'idéologie sportive sur celles de l'EP et de la gymnastique populaire. L'ainsi dite "pédagogie par objectifs » ne sera qu'un moyen supplémentaire de présenter comme innovant ce qui n'est que propagande et conditionnement pavlovien à l'idéologie sportive. Le paradoxe réside dans le fait que ce sont essentiellement des acteurs à gauche qui, voulant favoriser l'apprentissage du sport (démocratisation et massification) par le plus grand nombre, portent cette pratique originellement ancrée à une idéologie aristocratique ou grande bourgeoise (Citius, Altius, Fortius! Struggle for life), comme on l'a vu.

Les Instructions officielles de 1985, témoignent parfaitement de ce conflit en optant, comme toujours, pour une position médiane, neutralisante, conciliant la " pédagogie des conduites motrices", «les maitrises d'exécution » promues par les courants se situant dans le prolongement de la Ligue (dès lors disparue) et portés par Pierre Parlebast ${ }^{33}$ (proche alors des CEMEA) ou de manière plus radicale, sous la dénomination de Critique socio-politique du Sport par Jean-Marie Brohm ${ }^{34}$. Pour le premier, le sport n'est pas le seul moyen d'EP ; il est au contraire, par rapport aux jeux traditionnels, par exemple, «mathématiquement» pauvre, en termes de communication. Pour le second, il est socialement mauvais en n'étant que la réplique plus ou moins déformée du système capitaliste industriel. Le courant de la didactique des APS, lui, argumente non sur le fond 
idéologique mais sur la nécessité d'innover pédagogiquement en organisant une didactique rigoureuse.

71 Enfin, au tournant des années 1990, un nouveau débat éclate autour de trois visions antagonistes de l'activité physique en EP. La première, dans le prolongement de la pédagogie par objectifs et impliquant un certain nombre d'acteurs de la phase précédente, est favorable à une ingénierie didactique des activités physiques et sportives (Portes, Goirand, Marsenach, Amade-Escot, Pradet...), et considère que le sport est devenu la seule « activité culturelle de référence ». Il s'agit, là encore, de considérer que le sport est le seul support possible de l'EP parce que le sport est l'activité corporelle dominante. Ce courant sera dénommé « culturaliste » alors que, paradoxalement, il réduit la culture du corps au seul sport moderne, un peu comme si la littérature réduisait son programme au «nouveau roman». Encore une fois, le sport sous le couvert d'une nouvelle dénomination "activité culturelle de référence» ou "ingénierie didactique», est présenté comme innovant, alors qu'il s'agit toujours du même système de valeurs et de techniques (à quelques activités nouvelles près, californiennes ou autres) depuis la fin du XIX siècle.

Une deuxième vision, impulsée par l'inspection générale se centre sur une « didactique de l'Éducation Physique » (Delaunay, Pineau, Klein...). La publication d'une Introduction à une didactique de l'Éducation Physique ${ }^{35}$ par Claude Pineau et Michel Delaunay, en 1992, va tout à fait dans le sens des propositions précédentes de Pierre Seurin ou antérieurement de Paschal Grousset ou Georges Hébert. Il s'agit simplement de ne pas accepter la technique sportive (avec ce qu'elle suppose au plan idéologique) comme seule porteuse de l'EP. On cherche donc à faire émerger des "groupements d'activités" (cinq) non limités aux activités sportives et à la classification du Comité National Olympique et Sportif Français, généralement dominante (sept à huit groupements), ainsi que des «principes et règles d'action » puis des « principes opérationnels » qui cherchent davantage à se situer dans la transcendance de l'activité motrice de l'enfant que de la technique sportive stricte.

Mais, ici encore, en réalité, on trouve l'ancien conflit redistribué : Ligue vs Comité. Et ce qui sépare les deux camps réside bien dans le système idéologique fondateur. Le paradoxe est là encore très fort, puisque des acteurs plutôt marqués à droite (à l'inspection générale notamment) se retrouvent sur les bases idéologiques d'une "mystique de gauche » en soutenant, pour utiliser les mots du début du siècle : l'EP contre le Sport. Cependant cette fois, contrairement à la période des années soixante, la didactique de l'EP se pose, tout comme «l'ingénierie des activités culturelles de référence », en conception moderniste de l'Éducation Physique et Sportive. Il reste que le fond est toujours le même : les uns défendent la «technique » et l'idéologie sportive, les autres une idéologie de l'Éducation corporelle complète du plus grand nombre.

Une troisième conception "hédoniste" se construit autour de la revue Les Cahiers pédagogiques (Jacques André, Bernard-Xavier René...), Le Corps Enchaîné et Quel Corps? Ce groupe est une sorte d'hypertrophie extensive et pédocentrique de la critique du Sport. Très clairement il se situe dans le prolongement d'une vision du corps, libertaire, largement issue des évènements de 1968, mais aussi de visions beaucoup plus anciennes (hédonistes, cyniques, la mettriennes ou rabelaisiennes par exemple), où la « jouissance » du corps est première et où la contrainte technique peut être rejetée au profit du plaisir $\mathrm{du}$ jeu. Finalement, là encore, sont repris certains thèmes très pédocentriques pour lesquels l'enfant se construit d'abord en jouant et que l'on retrouvait dans la pensée de Grousset, mais aussi des Manuels d'exercices physiques et de jeux scolaires du début du siècle 
(1908). Cette fois pourtant, il s'agit de jeux d'enfants et non de jeux d'adultes comme le sport. C'est la mise de l'enfant au centre qui justifie l'existence de ce courant, mais il est finalement peu éloigné du courant précédent de la didactique de l'EP. Il s'en distingue toutefois par une vision extrêmement optimiste du développement de l'enfant en matière de technique du corps. Il suppose que les jeux, tous les jeux, suffisent à favoriser le développement corporel complet. Une scolarisation rigoureuse et une méthodologie très stricte ne semblent pas importantes pour les acteurs de ce courant. La paidéia de l'enfant est finalement suffisante.

Encore une fois, les textes officiels qui paraissent sous la dénomination «Programmes » en 1996 porteront la marque de ces trois tendances. Huit groupes d'activités satisfont le courant de la didactique des APS, des compétences générales et propres, celui de la didactique de l'EP, et une analyse de l'élève de sixième comme "agissant en vue d'une satisfaction et d'un plaisir immédiat ", celui des hédonistes. Mais, ici, à peine ce débat est-il entériné que surgit un renouvellement de ces programmes.

Un dernier conflit, aujourd'hui, toujours sous couvert d'innovation dans le champ, oppose ce qui est incorrectement appelé "courant développementaliste» et courant « culturaliste », alors qu'il s'agit toujours, simplement, des partisans du « sport éducatif » et «fini » pour les seconds et d'un modèle plus large de motricité ouverte pour les premiers. Le binaire originel du Comité (Culturaliste) et de la Ligue (Développementaliste), sous des formes conçues comme innovantes, est toujours présent. Et si le mot est dit la chose demeure toujours cachée, c'est-à-dire : une vision divergeant sur les principes, en terme de valeur.

Ces deux visions finissent pourtant par « s'accorder » (c'est, répétons-le, plutôt un « nondissensus ») sur un texte où les " compétences » - vision totalitaires pour certains (voir Le Goff, $1999^{36}$ - sont le centre de tout discours (c'est ce terme qui domine dans tous les programmes français à l'heure actuelle). Ce sont les programmes parus depuis 1999 pour les classes de $2^{\mathrm{e}}, 1^{\text {re }}$ et terminale. Cependant le fait que des programmes publiés en 1999 aient dû, sous la pression du courant "culturaliste», être abrogés et remplacés par d'autres (2000) où la place de la compétition sportive était plus marquée, montrent encore la puissance de la «mystique de droite » face à la «mystique de gauche», des résidus « archéologiques » du Comité, face à ceux de la Ligue.

\section{Conclusion}

Taire la chose et dire les mots, tel est le concept qui permet de donner une forte intelligibilité aux innovations dans le champ de l'EP.

En effet, ce sont, depuis l'origine étatique de l'institution, deux visions totalement antagonistes de l'exercice qui se sont mises en place: une vision "sportiste" (aristocratique et élitiste) et une vision " pédagogiste » (démocratique et égalitariste), ou pour le formuler autrement, une vision "matério-centrique» et une vision "puérocentrique ». Ces deux positions sont appuyées, à l'origine, sur le conflit radical au plan des principes et de l'idéologie politique que constitue la Commune de Paris (et peutêtre bien plus loin encore sous la $1^{\text {re }}$ République). D'elle, naissent deux institutions qui tairont, d'emblée leur origine politique: le Comité pour la propagation des exercices physiques (dit Jules Simon) et la Ligue Française d'Éducation physique. Et si, dès lors, les mots pourront à la surface être prononcés, la chose qui construit le fond : les barricades, 
le Mur des fusillés, la Semaine Sanglante, la condamnation de Grousset à l'exil par le gouvernement de Thiers, ne pourra plus jamais être dite. Il faudra donc, au cours du siècle, travestir le fond pour ne laisser apparaitre que la forme.

Sur cette base repose toute innovation en EPS au cours du siècle. À partir de son intégration dans les programmes, il faudra éviter de dire la chose, c'est-à-dire le Sport, celui-ci renvoyant axiologiquement et inexorablement au monde qui l'a fait naître et qu'il faut simuler de ne plus voir (pour des enseignants « républicains de gauche »). Il en va de même bien sûr pour la vision « communarde » de l'exercice.

Dès lors, innover en EPS consistera à cacher la chose derrière des mots qui pourtant conduisent tous au même objet. " Méthode sportive » vs «EP méthodique », " pédagogie par objectifs » vs " psychocinétique », «didactique de l'EP » vs «didactique des APS », « ingénierie didactique», «courant culturaliste» vs «développementaliste» ou " hédoniste ", " activité culturelle de référence », " essence de l'activité »... seront autant de termes qui viseront à ne pas dire la chose, à cacher l'indicible: un affrontement idéologique originel et fondamental, destructeur, parfois d'ailleurs à fronts renversés.

L'innovation pédagogique, en EPS, ne reste donc, bien souvent, qu'une affaire de règles et non une affaire de principes. Pourtant, chaque camp se croit tenu de reformuler l'identique en le faisant passer pour du neuf d'où une litanie de néologismes «didactiques» intelligibles pour le seul initié, d'où aussi, la force du conflit lors de chaque production d'innovation. Car, en effet, au travers de cette surface apparente, c'est un enjeu bien plus important qui se trame: valoriser pour tous les élèves de France en EPS, une vision élitiste ou une vision égalitariste de l'exercice. On voit bien, au cours de la période actuelle, ce que cela peut représenter d'enjeu de société.

\section{BIBLIOGRAPHIE}

BUI-XUAN G. et GLEYSE J. : L'Émergence de l'éducation physique. Georges Demenÿ et Georges Hébert. Paris, Hatier, 2001.

GLEYSE J. : Archéologie de l'Éducation physique au XXe siècle en France. Paris, PUF, 1995. GLEYSE J. : L'Instrumentalisation du corps. Paris, L'Harmattan, 1997.

GLEYSE J. : « Mystique de droite et mystique de gauche en pédagogie sportive, en France, sous la Troisième République », in International Journal of the history of sport, et STADION : «Le Sport en France de 1870 à 1940 », Sankt Augustin, Academia Verlag, pp. 125-139.

\section{NOTES}

1. ONFRAY M. : Politique du rebelle. Traité de résistance et d'insoumission. Paris, Grasset, 1999.

2. DEMENY G. : Les Bases scientifiques de l'éducation physique. Paris, Félice Alcan, 1902, p. 19.

3. TISSIÉ P.: l'Éducation physique au point de vue historique, scientifique, technique, pédagogique.... Paris, Larousse, 1901, p. 224.

4. FREDI de (baron de COUBERTIN) P. : L'Éducation en Angleterre. Collèges et Universités. Paris, 1888, p. 28, 324-326.

5. FREDI de (baron de COUBERTIN) P. : La Gymnastique utilitaire. Sauvetage - Défense - Locomotion. Paris, 1905, p. V.

6. FREDI de (baron de COUBERTIN) P. : Leçons de pédagogie sportive. Paris, 1921, p. 24. 
7. FREDI de (baron de COUBERTIN) P. : Leçons. Paris, p. 12.

8. FREDI de (baron de COUBERTIN) P.: «Discours de la cérémonie d'ouverture des Jeux Olympiques d'Hiver ", Chamonix, 5 février 1924, in Message Olympique, 32, 1992, p. 5.

9. FREDI de (baron de COUBERTIN) P. : Leçons de pédagogie sportive. 1921, p. 45.

10. DARYL P. : «Les sports anglais »,in Le Temps, 16 Février 1890, Paris, p. 10.

11. BULLETIN OFFICIEL du $1 »$ août 1923, p. 135.

12. BULLETIN OFFICIEL du l' :r août 1923, p. 82.

13. Ibid

14. LOISEL E. : Les Bases psychologiques de l'Éducation physique. Paris, Bourrelier, 1955, p. 139.

15. Ibid., p. 3.

16. Ibid, p. 24.

17. Ibid

18. Ibid., p. 206.

19. BULLETIN OFFICIEL du 23 août 1938, p. 37.

20. Md.

21. BAQUET M. : Éducation sportive. Initiation et entraînement. Paris, Godin, 1942.

22. BAQUET M. : Précis d'initiation sportive. Paris, Godin, 1943.

23. Par ailleurs mort pour fait de résistance en 1943.

24. BAQUET M. : Éducation sportive. Initiation et entraînement. Paris, Godin, 1942, p. 11.

25. BAQUET M. : Ibid., p. 15.

26. DE RETTE J. : « La gymnastique de grand père est MORTE », in EPS, 61, juillet 1962, p. 20-26.

27. TESSIÉ J. : « Essai d'une systématique des sports », in EPS, 37,1956, p. 34.

28. SEURJN P. : Vers une Éducation physique méthodique. Paris, FFGE, 1949, p. 16.

29. Instructions officielles ministérielles du 19 octobre 1967, p. 1.

30. Instructions, p. 3.

31. KLEIN G. : « Eléments de réflexion et de transmission sur le procès d'institution d'une culture corporelle : le cas de l'éducation physique scolaire », in Tréma, Spécial Hors Série № 1, 1997, p. 33-47.

32. LE BOULCH J. : Face au sport. Paris, ESF, 1977.

33. PARLEBAS P. : Lexique commenté en sciences de l'action motrice. Paris, ENSEP, 1981.

34. BROHM J.-M. : Sociologie politique du sport. Paris, Delarge, 1977

BROHM J.-M. (dir.) : Quelles pratiques corporelles maintenant ? Paris, Delarge, 1978.

35. PINEAU C. et DELAUNAY M. : Introduction à une didactique de l'Éducation physique et sportive. Paris, EPS, 1992.

36. LE GOFF J.-P. : La Barbarie douce. La modernisation aveugle des entreprises et de l'école. Paris, La Découverte, 1999.

\section{RÉSUMÉS}

Deux «mystiques» divisent à l'origine le champ des pratiques corporelles scolaires, non sans paradoxe dans l'innovation en Éducation physique. D'un côté, l'éduqué physique: vision égalitariste, tournée vers le plus faible (Ligue d'Education physique de Paschal Grousset). De l'autre le sportif: vision élitiste, tournée vers le plus fort (Comité «Jules Simon» et baron de Coubertin). Ces visions antagonistes sont depuis la fin du XIX» siècle un pivot des débats. Mais 
une deuxième logique intervient: le XXe siècle sera aussi une redécouverte permanente du rousseauisme ou des méthodes actives.

From the outset, two "ideals" are opposed one to the other in the field of school physical activities. On the one hand, the "educated physically fit", reflecting an egalitarian vision focused on the least physically capable individual. On the other hand, the "sportsman", reflecting an elitist vision focused on the physically strongest. These antagonistic ideals are the crux of debates sine the end of the XIXth century. But the XXth century means an alternative to this dichotomy with the recurring rediscovery of Rousseau and activity centered methods.

\section{INDEX}

Mots-clés : éducation physique, innovation, méthode active, mystiques, récurrence, sport

Keywords : activity centered method, mystique, physical education

\section{AUTEUR}

\section{JACQUES GLEYSE}

IUFM de Montpellier, Didaxis-Dipralang et Corps \& Culture, Université de Montpellier I 